\title{
Desarrollo de competencias en educadores físicos en formación a través de la inclusión de programas de educación aventura en México Development of competencies in physical education teachers in training through the inclusion of adventure education programs in Mexico \\ *Ramón Alfonso González-Rivas, *M aría Del Carmen Zueck Enríquez, **Antonio Baena-Extremera, \\ *Rigoberto M arín U ribe, *Ma. Concepción Soto Valenzuela, *Hugo Eduardo Irigoyen Gutiérrez *U niversidad Autónoma de Chihuahua (M éxico), **U niversidad de Granada (España)
}

\begin{abstract}
Resumen: El objetivo de este estudio fue identificar las competencias profesionales desarrolladas por profesores de educación físicaen formación, durante la aplicación de actividades en el medio natural. M étodo, un estudio de caso con nueve estudiantes de licenciaturaen educación física, solo dos de ellos con experiencias previas en actividades en el medio natural. Seempleó una secuencia didáctica basada en la metodología de Educación deAventura. La recolección de datos se llevó a cabo por medio de videograbación, el análisis se realizó utilizando elementos de la teoría fundamentada a través del software Atlas Ti. Los resultados revelaron cuatro competencias profesionales favorecidas durante la aplicación de la secuencia didáctica: 1) trabajo en equipo y liderazgo, 2) dominio conceptual de Educación Aventura, 3) dominio técnico de actividades lúdicas en el medio natural, y 4) pedagogía y didáctica de la Educación Física en actividadesal aire libre; adicional mente se encontró que durante las actividades los hombres fueron más participativos que las mujeres. Se logró identificar competencias que no estaban consideradas en la planeación académica docente, llevando a concluir que los programas de Educación Aventura pueden fortalecer la formación de los estudiantes de educación física.
\end{abstract}

Palabrasclave: Educación Física, educación de aventura, actividadesfísicas en el medio natural, secuenciadidáctica, pedagogía y didáctica.

\begin{abstract}
The purpose of this study was to identify the professional competencies developed by physical education teachers in training, during the application of outdoor activities. Method, a case study, participated werenine undergraduate studentsin physical education, two of them had previous experiences in outdoor activities. A didactic sequence based on theAdventure Education methodology was used. Data was collected by video recording, and the analysis of the data obtained was processed by the elements of grounded theory through the Atlas Ti software. The results showed four professional competencies favored during the application of the didactic sequence: 1) teamwork and leadership, 2) conceptual domain of Adventure Education, 3) technical domain of recreational outdoor activities, and 4) pedagogy and didactics of the physical education in outdoor activities; additionally, it was found that mal es participated more than femalesduring the activities. It wasal so possible to identify competencies that were not considered in the academic planning of teachers, leading to the conclusion that Adventure Education programs can strengthen the training of physical education students.
\end{abstract}

Keywords: Physical education, adventure education, physical activities in the natural environment, didactic sequence, pedagogy and didactic.

\section{Introducción}

La universidad ha evolucionado, pasando de ser el vínculo para la obtención de conocimiento a convertirse en el sitio idóneo para desarrollar competencias profesionales, que permitan a los egresados un desenvolvimiento oportuno en una sociedad cambiante. La Decla ración de Bolonia en 1999, dio como resultado cambios significativos en laeducación universitaria, principalmente model os educativos basados en competencias (Losada \& García, 2018). La competencia es definida como la

Fecha recepción: 02-11-20. Fecha de aceptación: 10-03-21

Ramón Alfonso González-Rivas

rgrivas@uach.mx manera en que el sujeto resuelve una situación determinada, paralo cual debe poner en práctica conocimientos, habilidades y actitudes (Guzmán \& M arín, 2011). En México, los programas educativos de formación en Educación Física (EF), deporte y recreación en educa ción superior, orientan sus perfiles de egreso a tendencias tradicionales del área como son la educación y el entrenamiento deportivo (Valencia, Salazar, Lozano, Figueroa \& Flores, 2012). Los profesores de EF en formación requieren fortalecer competencias didácticascon un enfoque práctico, y de esta forma poder vivenciar las competencias docentes que deberán aplicar cuando egresen (Hernández-Amezcua \& M artínez-Flores, 2017).

En la EF, las actividades físicas en el medio natural 
(AFM N) son una tendencia emergente (Marinho, Santos, M anfroi, Figueiredo \& Brasil, 2017). En este sentido, la Educación deAventura (EA) es un modelo peda gógico basado en el aprendizaje experiencial, sólido y práctico que involucraalos estudiantesen retos grupales e individuales por medio de actividades físicas, diferentes a los modelos tradicionales (Lund \& Tannehill, 2014; Sutherland \& Legge, 2016). En laEA se plantean diversas actividades en las que el sujeto interacciona con el entorno natural y pueden incluir elementos de riesgo real 0 aparente, donde se involucran diversos factores como la experiencia y habilidades de los participantes en distintas circunstancias (Gilbertson, Bates, McLaughlin \& Ewert, 2006). Por su parte, Caballero, Hernández-Hernández y Reina (2018), añaden que son actividades de carácter lúdico-recreativo y que favorecen el desarrollo personal y social. De acuerdo con Doering (2006), el enfoque de la EA consiste en dar al estudiante experiencias de aprendizaje que le permitan explorar y construir sus conocimientos del mundo real. Estos programas contribuyen a fomentar el trabajo colaborativo entre pares por medio de actividades de solución de problemas, en donde cada integrante tiene una responsabilidad (O rson, M cGovern, \& Larson, 2020).

Según la literatura científica, los programas de EA ayudan a alcanzar múltiples beneficios, como el aumento en la actividad física (Li, Chung $\&$ Ho, 2013), disminuyen los síntomas depresivos y ansiedad (Li, Chung, Ho, Chiu \& Lopez, 2013), incrementa la sociabilidad y el control emocional (Stuhr, Sutherland, Ressler \& 0 rtiz-stuhr, 2015), aumento de satisfacción y autoestima en los participantes (Baena-Extremera, Granero-Ga Ilegos \& O rtiz-Camacho, 2012), estimula la resiliencia en adolescentes (Scarf, Hayhurst, Riordan, Boyes, Ruffman \& Hunter, 2017), se alcanzan beneficios físiCOS, cognitivos y en el autoconcepto (W illiams \& Wainwright, 2016). En este sentido, la EA puede tener un papel importante en el aprendizaje (Lee $\&$ Zhang, 2019). Entendiendo la EA como un modelo pedagógico emergente (Fernández-Río, Calderón, Hortigüela, Pérez-Pueyo \& Aznar, 2016), tiene la flexibilidad de hibridarse con otros modelos (Lamoneda, GonzálezVíllora \& Fernández-Río, 2020).

Alrededor del mundo, se han implementado los programas de EA en la formación académica de profesionales en el área de EF, deporte y recreación. En España, Caballero, Velo y Hernández (2020), encontraron que la inclusión de elementos de aventura en el contexto universitario contribuyó a la formación profesional de los estudiantes, favoreciendo el desarrollo de competencias específicas. En Brasil, M arinho et al. (2017), aplicaron programas de EA desarrollando competencias sociales, personales, éticas y específicas que fueron significativas en la formación de universitarios. En Inglaterra, Leather y N icholls (2016) encontraron que la aplicación de EA por parte de los estudiantes en la comunidad permitió el desarrollo de empatía y conexión con la natural eza.

Uno de los modelos de EA más exitosos ha sido la propuesta de Rhonke (1989) que se caracteriza por la utilización de actividades y situaciones con cuerdas a cierta altura. En este sentido, las actividades con cuerdas, los puentes arbóreos (principalmente puente de mono y puente tibetano) han sido utilizados en diversos programas de actividad física, evidenciando potencial lúdico y pedagógico (Escaravajal-Rodríguez, BaenaExtremera, Ayala-J iménez \& Ruiz-Fernández, 2017; Hurtado-Barroso, Sanabrias-M oreno, Sánchez-Zafra \& Cachón, 2020). Para poder realizar un puente arbóreo es indispensable conocimiento básico en cabuyería, entendiendo ésta como el arte o habilidad de realizar y aplicar distintos tipos de nudos (Rubio \& Rico, 2012).

Las universidades tienen la tarea de formar profesionales competentes para afrontar los retos de la actual idad. Sin embargo, en las carreras de EF parece existir la necesidad de sincronizar los planes y programas de estudio con la realidad que afrontaran los egresados (N ascimento, Ramos, Marcon, Saad \& Collet, 2009). La educación superior, particularmente los planes de estudio en EF tienen la responsabilidad de transformar contextos, lo que conlleva a desarrollar competencias vanguardistas, gestión innovadora del conocimiento, así como atender los intereses profesionales de los estudiantes (Badau, 2016; Sáez-Padilla, Tornero \& Sierra, 2017). En esta misma lógica, existe una expansión de actividades físico-recreativas en la búsqueda de tendencias modernas, que pueden ser una alternativa viable. Por tal motivo, los currículos de las universidades deben considerar contenidos AFMN utilizando la EA, en la actualización de sus programas (Badau, 2017).

En México, las AFM N emergen como una posibilidad de trabajo innovadora para el profesorado de EF y deben ser contenidos de interés en la formación de aca démica (González, Zueck, Marín \& Blanco, 2019). Específicamente, la licenciatura en EF de la U niversidad Autónoma de Chihuahua (UACH), en su plan de estudios del 2005 y con vigencia en el momento que se realizó este estudio, contempla competencias básicas, profesionales y específicas. Las competencias básicas son 
comunicación, solución de problemas, trabajo en equipo y liderazgo y emprendedor. Las profesionales son cultura de la salud, elementos conceptuales básicos de salud, principios docentes e investigación en salud. Por último, las específicas, EF, deporte y recreación, enfoque psicopedagógico de la EF, deporte y recreación, y fundamentos biológicos de la EF del deporte y la recreación. Es importante destacar que, en los primeros tres años de formación, no se contempla contenidos de AFMN (Zueck, Guerrero \& Blanco, 2009).

Como se puede leer en líneas anteriores, los programas de EA tienen un potencial educativo muy importante y pertenece al campo de acción de la EF. Por tal motivo, en esta investigación se tiene como objetivo identificar las competencias profesionales desarrolladas por profesores de EF en formación durante la aplicación de actividades en el medio natural. Dicho lo anterior, este estudio es un primer acercamiento a la posible inclusión de contenidos deAFMN con metodologías como EA, en la formación académica del profesorado.

\section{Método}

El presente estudio parte del paradigma interpretativo (Maldonado, 2018), utilizando el método estudio de caso. Este método se define como el análisis y descripción holística e intensiva de un fenómeno específico, como un programa, un evento, una persona, un proceso, una institución, comunidad o grupo de personas (Merriam, 1998). Se optó por esta metodología, porque permite conocer e interpretar los acontecimientos. Reforzando esta idea, paraY in (1994), el estudio de caso es pertinente cuando el fenómeno a investigar es analizado en su contexto natural, sin al terar al guna va riable y en donde los resultados son producto de factores cotidianos.

\section{Participantes}

El caso de estudio estuvo conformado por el grupo de estudiantes del área curricular de recreación, en la asignatura de juego y EF que se imparte en cuarto semestre en la licenciatura en EF de la UACH. Distribuido por nueve estudiantes de 19 y 20 años, seis hombres y tres mujeres, dos de ellos con experiencias previas en actividades con cuerdas.

También participaron dos expertos en investigación educativa, evaluación de programas y competencias aca démicas, su colaboración validó el proceso de codificación y categorización de resultados. A continuación, se presenta brevemente su experiencia:
Experto 1. Con Doctorado en Ciencias de la Cultura Física, docente en la UACH, miembro de Sistema Nacional de Investigadores nivel 1, cuenta con 30 artículos publicados en revistas de impacto, coautor del libro: Reforma curricular de la licenciatura en educación física: Evolución histórica al 2008.

Experto 2. Con Doctorado en Ciencias de la Cultura Física, docente en la UACH, directora de más de 100 tesis terminadas de nivel maestría y licenciatura; además en tesis de doctorado en desarrollo, con más de 25 artículos publicados en revistas de impacto.

\section{Instrumentos}

Para conocer la percepción y competencias desarrolladas por los estudiantes se utilizó la observación no participativa, definida como una técnica en la que investigador se limita únicamente a registrar los hechos que son de su interés sin participación en los acontecimientos, en al gunos casos permite enfocarse en el objeto de estudio sin que éste se dé cuenta de ello (Campos \& Martínez, 2012).

Se empleó la videograbación, entendida como la herramienta que permite al investigador aproximarse al objeto de estudio, justo como ocurre naturalmente. En la videograbación, se tiene la oportunidad de ver las veces que sea necesario una misma situación (O rellana \& Sánchez, 2006). Los investigadores pueden observar la videograbación en un ambiente controlado y de forma colaborativa para poder realizar el análisis cualitativo, lo que da certeza de confiablidad interna a la investigación (Stigler, Gonzales, Kawanaka, Knoll \& Serra no, 1999).

Se utilizó el software Atlas Ti en su versión 7.5, herramienta ha demostrado ser una eficaz para el análisis hermenéutico de datos en estudios del contexto educativo (San Martín, 2014).

\section{Análisis de datos}

Para el análisis de datos se utilizó las bases de la teoría fundamentada, específicamente el diseño emergente, que se caracteriza por realizar la codificación abierta de los datos, para así, crear categorías (G laser, 1992), por lo que es un proceso inductivo. Se optó por este diseño ya que las categorías surgen de los mismos datos, en este sentido, no se contemplaron categorías prestablecidas.

\section{Procedimiento}

Se obtuvo el consentimiento de la autoridad académica de la institución educativa para realizar el estudio. 
Previamente, se llevó a cabo una etapa diagnóstica del desempeño docente que incluyó entrevistas con estudiantes y docentes, así como observaciones de clase, para identificar necesidades de capacitación y actualiza ción de los docentes en el rubro de recreación. Los ha llazgos revelaron la necesidad de capacitar a los profesores desde las perspectivas pedagógica y disciplinar, incluyendo entre otros temas, los programas de EA. Precisamente, el docente universitario que participó en este estudio fue parte de dicha capacitación. Con la finalidad de innovar su asignatura, el docente diseñó una secuencia didáctica con contenidos deAFM N utilizando la metodología de EA: cabuyería y puentes arbóreos, esta secuencia se aplicó con sus 9 estudiantes de la asignatura de juego y educación física.

La secuencia didáctica se dividió en dos sesiones de una hora con 40 minutos cada una. Previo a la primera sesión el docente encargó a los estudiantes realizar una búsquedabibliográfica sobreAFM N y programas de EA. En clase presencial se social izó y debatió sobre la aplica ción de EA en la EF. Por medio de preguntas del docente, los estudiantes mencionaron sus experiencias previas en AFM N, favoreciendo el conocimiento individual y grupal. Para tal efecto, el docente hizo las preguntas: ¿En qué actividades de aventura han participado? ¿Alguno de ustedes ha escalado, ido de campamento o tienen experiencia en actividades de aventura?

Para la siguiente actividad, el grupo se dividió en equipos de tres, cada estudiante tuvo una cuerda de un metro de longitud, el docente envió por W hatsApp enlaces de videos en donde se explicó cómo realizar los nudos de siete, ocho, pescador y leñador. Los alumnos trabajaron en tercias, para de forma práctica y colaborativa lograr aplicar correctamente la cabuyería. En todo momento el profesor aclaró dudas, la actividad anterior estimuló el conocimiento básico para la utilización del material. La sesión culminó con la reflexión individual y grupal respecto a las posibilidades didácticas de cabuyería en EF. En este sentido, el docente preguntó ¿De qué manera podemos utilizar la cabuyería en la EF?

La segunda sesión se desarrolló en un espacio al aire libre, con suficientes árboles, el docente explicó la metodología para montar un puente arbóreo, así como el tensado de la cuerda. El grupo se dividió en equipos de tres estudiantes, cada triada hizo un puente arbóreo y tuvo la libertad de hacer las modificaciones que consideraron pertinentes para que fuera un desafío motriz, cognitivo y emocional. Para continuar con la experiencia de aventura, los estudiantes cruzaron por los puen- tes realizados por sus compañeros, y cada estudiante se aseguró que los otros integrantes de su triada concluyeran cada reto.

Por último, lasesión concluyó con unaautorreflexión y reflexión grupal sobre las sensaciones y retos al momento de cruzar por los puentes, así como, sobre las posibilidades pedagógicas y aplicación de los puentes arbóreos en el futuro profesional. Para tal efecto, el docente utilizó las siguientes preguntas generadoras: ¿Q ué utilidad tienen los puentes arbóreos en las clases de EF? ¿Q ué experiencia tuvieron al momento de pasar a través del puente? En cuanto a los nudos y el tensado de la cuerda ¿Se les hizo difícil? De esta forma se aplicaron elementos de las fases de programas de EA propuestos por BaenaExtremera (2011).

Para la recogida de datos, previo consentimiento de estudiantes y el docente, las dos sesiones en las que se aplicó la secuencia didáctica fueron videograbadas y posteriormente transcritas en formato RTF. Después, utilizando el diseño emergente y por medio de software Atlas Ti, se realizó el proceso de codificación y categorización, este proceso fue validado por los dos expertos en investigación educativa.

\section{Resultados}

Una vez realizado el análisis cual itativo, se lograron identificar cuatro categorías (competencias) que favorecieron la formación académica del profesorado de EF. Destaca que se identificó una competencia del plan de estudios: trabajo en equipo y liderazgo. Sin embargo, se encontró tres competencias ajenas a dicho plan. En la tabla 1, se expone la competencia y los códigos que le dan sustento.

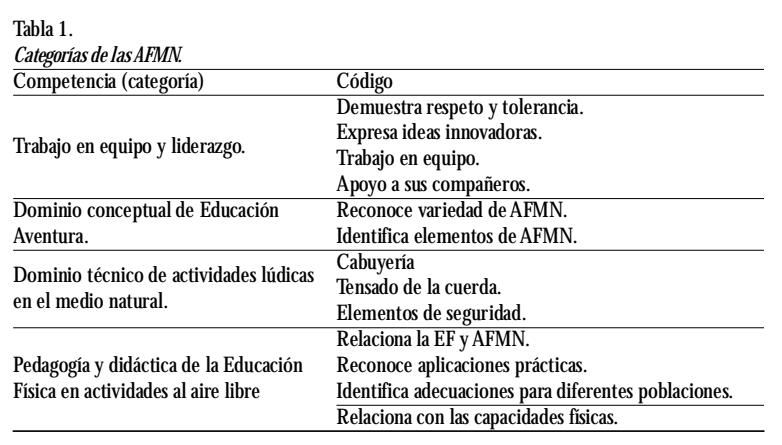

\section{Trabajo en equipo y liderazgo}

Durante la actividad de cabuyería hubo dos estudiantes con experiencias previas, sin que el profesor lo solicitara, estos estudiantes apoyaron a los compañeros que tenían problemas con el ajuste de los nudos. En la actividad de los puentes arbóreos, en lo referente al 
tensado de la cuerda los estudiantes debatieron y analizaron en grupos la técnica del tensado, evidenciando procesos de reflexión individual y grupal. Para lo cual, durante la secuencia didáctica, los estudiantes estimula ron actitudes y val ores como la ética, liderazgo, inicia tiva, respeto y tolerancia, favoreciendo el trabajo en equipo.

\section{Debate en clase:}

... yo creo que la cuerda se puede tensar de muchas formas, se ata la cuerda a un árbol o poste, en otro árbol se coloca el anillo y se le coloca un tensor... entonces el otro extremo de la cuerda se amarra al tensor... [E3]

... pero tienes que ser muy exacto para poner el tensor... y aparte el tensor sí es más caro que así con los mantillones... E6]

... pues sí, pero está más fácil, que aprenderse todos los nudos y con el tensado en block yo me reburuje mucho... [E3]

Comentario de apoyo durantela actividad y reflexión al final de la sesión:

... a ver ¡cómo no vas a poder! agárrate bien de la cuerda de arriba, hazte para atrás, acuéstate... pero no sueltes le de arriba, es la de seguridad, ándale así está bien, ya solo avanza... [E7]

... yo vi mucho trabajo en equipo, al momento de colocar lo puentes y en el desarrollo de las actividades, para mí también fue importante ver y escuchar las sugerencias de mis compañeros, sobre todo porque yo nunca había hecho una actividad así...

\section{Dominio conceptual de EA}

Los estudiantes lograron identificar y relacionar ca racterísticas propias de la EA, como estimular confianza, adrenalina y ser un desafío, evidenciando comprensión y entendimiento del modelo pedagógico.

Reflexiones al final de la sesión:

... hay que tener confianza en uno mismo y en los compañeros porque al principio daba miedo, creo que esas con las características que vimos la clase pasada... [E1]

... con los ojos tapados yo sentí mucha adrenalina, pero de eso se tratan estas actividades, por eso se llama Educación Aventura... [E3]

... para mí, todas las actividades fueron un desafío, aunque no estaba muy alto, no sentía seguridad al ir avanzando sobre la cuerda, como dice Adrián, de eso se trata esta actividad... [E7]

\section{Dominio técnico de actividades lúdicas en el medio natural.}

Los estudiantes mostraron interés en la utilización de cuerdas con fines lúdicos, dos de ellos tenían conocimiento previo sobre cabuyería, pero no sobre los nudos específicos necesarios para elaborar puentes arbóreos (siete, ocho, leñador y pescador). Sin embargo, el conocimiento previo de la cuerda les facilitó aprender los nuevos nudos. El nudo de siete fue el más complicado para los estudiantes, en contraparte el nudo de leñador fue el más sencillo. En relación con la elaboración del puentearbóreo, los estudiantestuvieron dificultadescon el tensado (en block). Sin embargo, con el auxilio del docente lograron desarrollar las competencias necesarias.

Comentario de estudiante durante le sesión:

... me imagino que siempre es recomendable que ya haya al guien detrás del compañero de la cuerda no se vaya a caer...

[E2]

... profesor, usted qué opina con esta cuerda (la de abajo), yo la siento muy floja, se va a bajar mucho cuando alguien se suba sobre ella... entonces la aprieto más... (alumno ajusta la cuerda) ahí está, creo que ya quedo mejor... [E7]

... yo creo que la cuerda de arriba esta floja... puede ser peligroso, déjemela aprieto más... [E4]

Comentarios reflexivos al final de la sesión:

... cuando comenzamosa hacer lasactividades de cabuyería no entendía su aplicación, hasta que hicimos los puentes, el nudo de siete es el que se me hizo más difícil, los otros sí los puedo hacer... [E1]

... lo de los nudos se me hizo fácil, aunque la verdad ya conocía algunos, el único nuevo para mí fue el de siete... 10 quesí mellamó la atención fue el tensado de la cuerda, no sabía nada, pero si lo entendí bien... [E2]

... aquí en el grupo, si pude hacer mi puente, pero con la ayuda del profesor y mis compañeros, es cuestión de práctica... E7]

... lo más complicado fue el nudo de siete y el tensado de la cuerda... sí lo hice, pero necesito practicarlo más... [E9]

\section{Pedagogía y didáctica de la Educación Física en actividades al aire libre.}

El puente de mono fue el primero realizado por los estudiantes. A petición del docente, reflexionaron sobre posibles variantes, con este mismo puente. El profesorado en formación sugirió modificaciones para incrementar el grado de dificultad.

Comentarios durante la sesión:

... pues cuando yo me subí, la cuerda de arriba estaba muy floja y se me hizo muy difícil mantener el equilibrio al avanzar... yo creo que para hacerlo más difícil también se puede aflojar las cuerdas... [E4]

... pues hacer el recorrido, pero de espaldas o con los ojos vendados, está más complicado... otra variante que se me ocurre es pasar por parejas y que en al gún punto se tengan que 
cruzar... [E5]

... otra variante podría ser usando solo una mano y en la otra traer una pelota y queno secaiga, pero eso sería para niños que ya tengan más habilidad... [E7]

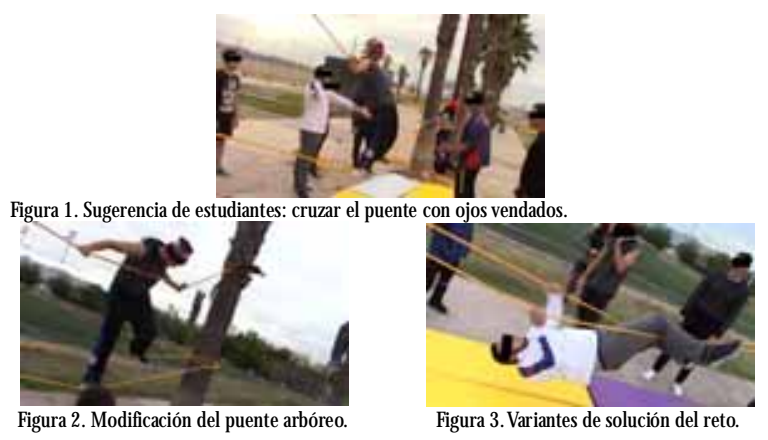

Los estudiantes propusieron hacer una modificación en el diseño del puente de mono, consistió en incluir una cuerda al mismo nivel de la cuerda inferior. Se evidenció la creatividad y capacidad de adecuación de los estudiantes, pero ahora desde la perspectiva del diseño del puente, lo que favoreció competencias pedagógicas y didácticas específicas. Surgieron diferentes maneras de cruzar por el nuevo puente (figuras 2 y 3 ).

Comentarios durante la sesión:

... colocando otra cuerda a la misma altura de la inferior, sería interesante porque habría más maneras de pasar por el puente... [E6]

... otra opción podría ser, al contrario, es decir, poner dos cuerdas arriba y solo una abajo, aunque creo que eso sería muy fácil porque habría dos cuerdas para sujetarse... [E7]

Los estudiantes fueron conscientes de que las actividades de EA son edificantes en su formación profesional y con aplicabilidad en su futuro trabajo. Lograron vincular la actividad de puentes arbóreos con elementos de la psicomotricidad, capacidades físicas y el impacto psicológico, así como, identificar la población con quienes podrían aplicar las actividades.

Reflexiones al final de la sesión:

... pues a mí me gustó, es al go diferente a los clásicos jugos que se pueden poner en una escuela, son divertidos, yo creo que a niños de quinto o sexto de primaria les gustarán mucho... [E4

... con estas actividades se pueden desarrollar muchas habilidades motrices, como fuerza, equilibrio y coordinación... creo que estas se pueden aplicar a personas de cualquier edad, no solo para niños... [E5]

... yo pensé que era más fácil, pero no, se tiene que hacer fuerza en los brazos y hay que tener mucho equilibrio... inclusive para adolescentes 0 personas de universidad pueden ser atractivos... [E7

Al momento de analizar la videograbación, se en- contraron un hallazgo que llamó la atención. Durante las actividades los hombres fueron participativos, en contraparte las mujeres adquirieron una postura pasiva.

\section{Discusión}

El objetivo de esta investigación fue identificar las competencias profesionales desarrolladas por profesores de EF en formación durante la aplicación de actividades en el medio natural. La importancia de este estudio radica en conocer dichas competencias para que en un futuro sean incluidas en el plan de estudio de educadores físicos en la UACH.

Los resultados apuntan hacia el desarrollo de la competencia de trabajo en equipo y liderazgo (competencia que se encuentra establecida en el plan de estudios), que se manifestó por medio de actitudes y valores en favor de habilidades sociales como cooperación y apoyo. Así mismo, en momentos puntuales de la sesión, el profesorado en formación debatió ideas bajo un marco de tolerancia y respeto. En esta misma línea se encuentran los resultados de M ediavilla, Gómez y Gómez (2020a), ellos aplicaron un cuestionario antes y después de realizar AFM N, encontrando aumento en las relaciones interpersonales en los participantes, favoreciendo la cohesión grupal en $10.72 \%$. En una investigación similar, Mediavilla, Gómez, Martín y Gómez (2020b) encontraron que el $81.7 \%$ de los estudiantes señalaron haber desarrollado competencias pararelacionarse mejor con sus compañeros. Estos resultados, se sitúan en la misma línea a los de Caballero et al. (2020), Marinho et al. (2017), Richmond, Sibthorp, Gookin, Annarella y Ferri (2018), Stewart, Carreau y Bruner (2016). En este sentido, es evidente que realizar actividades físicas con fines académicos en el medio natural contribuyen a una mejor relación entre los estudiantes, sin embargo, paraqueesto sucedaesfundamental unabuena planeación de actividades.

Se encontraron tres competencias no establecidas en el plan de estudios, hallazgo muy importante que sugiere la actualización de programas analíticos en el área curricular de recreación, en este sentido se logrará contribuir en el desarrollo de competencias de los estudiantes desde AFM N. Para la adquisición de estas nuevas competencias fue relevante el aprendizaje por medio de la experiencia, al igual que en con las investigaciones de Caballero et al. (2020) y Marinho et al. (2017). En esta misma línea, la totalidad del profesorado en formación que participó en el estudio de Granero 
(2015), valoraron favorablemente el hecho de que las actividades de aprendizaje se realizaran en el medio natural, que fueron presenciales y prácticas. Mediavilla et al. (2020b), en su investigación encontraron que el 92.5\% de los estudiantes expresaron haber adquirido conocimientos después de sesiones en el medio natural. Por su parte, Mediavilla et al. (2020a), utilizando aprendizaje experiencial, encontraron que los estudiantes aumentaron en un $29.6 \%$ sus conocimientos. En esta misma línea se encuentran los resultados de otros estudios (Agostini, Minelli \& Mandolesi, 2018; Gleason \& Rubio, 2020). En la investigación de Mackenzie, Son, y Eitel (2018), identificaron un aumento significativo en variables de autonomía y desarrollo de competencias utilizando actividades de aventura al aire libre en contraste con metodologías tradicionales. De acuerdo con Navarro-Patón, Rodríguez y Rico-Díaz (2019), en la formación de educadores físicos aprender por medio de la experiencia trae consigo satisfacción en el estudiante, debido a que percibe autonomía para desempeñar una función específica. La coincidencia de estos resultados sugiere que actividades de aprendizaje en el medio na tural, favorecen la adquisición de conocimientos. Sin embargo, se recomiendatener precaución, evitando que las actividades se conviertan en una experiencia única mente lúdica, para que realmente exista aprendizaje significativo debe haber periodos de reflexión y permitir autonomía al estudiante.

En esta investigación, se observó que la mayoría de losparticipantessemostraron participativos. Deacuerdo con Barrable y Lakin (2019), existe relación entre la enseñanza en el medio natural y la motivación por el aprendizaje. En esta misma línea, se sitúan los resulta dos de Marinho et al. (2017), al mencionar que motiva ción es otro factor que puede influir en el proceso de aprendizaje. Para los estudiantes, practicar actividades con materiales novedosos y en escenarios distintos a los tradicionales, son experiencias enriquecedoras en su formación académica (Fuentesal-García \& ZamoranoSande, 2021). En este sentido, los puentes arbóreosfueron de interés para el profesorado en formación, al gunos los consideraron innovadores, atractivos y de gran relevancia en su futuro profesional, hallazgos similares a los de Badau (2017). La concordancia de estos resultados sugiere que las AFM N, en este caso cabuyería y puentes arbóreos, estimulan la participación de los estudiantes, lo que favorece el desarrollo de competencias profesionales.

Con respecto a que en este estudio las mujeres mostraron una actitud pasiva durante el desarrollo de las actividades; M artín y M ediavilla (2020) encontraron diferencias significativas entre el género y la realización de AFMN, siendo las mujeres quienes menos las practican. Resultados congruentes con la investigación deWarren, Mitten, D'A morey Lotz (2019), dondeidentificaron que los programas de EA tienen un currículo oculto que conduce a valores, actitudes y prácticas que dejan en desventaja a las mujeres. Lo anterior, sugiere que las mujeres tienen resistencia a actividades poco conocidas, donde hay cierto nivel de riesgo controlado y dosis de adrenal ina, a pesar de ello, es importante no generalizar esta aseveración. En este mismo sentido, se considera prudente desarrollar programas de AFMN, con metodologías más inclusivas para las mujeres.

Los resultados encontrados revelan la necesidad de fortalecer la formación de los estudiantes con plantea mientos curriculares, soportados con infraestructura y materiales como recursos pedagógicos que permitan desarrollar aprendizajes orientados a su formación en el área de recreación, particularmente la EA, como es el caso (Baker \& O 'Brien, 2019; Dalmau, Jiménez, Gómez \& Gargallo, 2019; Kalungwizi, Krogh, Gjøtterud \& Mattee, 2018; Mackenzie, et al. 2018; Navarro, Arufe \& Sancosmed, 2015; W illiams \& Wainwright, 2019). Desar rollar estas competencias representa la oportunidad de trabajo en el ámbito del ocio, recreación y turismo (González, et al., 2019; Pérez, Vilanova \& Grimaldi, 2016), o de emprendimiento (Sánchez, García, Grimaldi $\&$ Feria, 2017), lo que diversifica el campo laboral de los educadores físicos.

\section{Conclusiones}

En esta investigación se ha tenido un primer acerca miento a la introducción de AFM N utilizando la metodología de EA en la formación de profesores de EF en la UACH. En congruencia con el objetivo de esta investigación se logró identificar una competencia ya establecida en el plan de estudios, tres más que surgieron de este estudio y que contribuyen a la formación del profesorado. A continuación, se definen las tres competencias:

Dominio conceptual de Educación Aventura: reconoce características conceptuales de los programas de Educación Aventura.

Dominio técnico de actividades lúdicas en el medio natural: utiliza diversos recursos materiales con fines lúdicos en actividades físicas en el medio natural.

Pedagogía y didáctica de la Educación Física en actividades al aire libre.: diseña y adapta actividades 
innovadoras con fines pedagógicos en el medio natural.

Se considera importante la aplicación de contenidos relacionados aAFM N en la formación de profesores de $E F$, puesto que este tipo de actividades ofrecen alternativas pedagógicas con importantes beneficios, así como, oportunidades de trabajo.

El estudio no estuvo exento de limitaciones, se analizó una secuencia didáctica que contemplo únicamente contenidos de cabuyería y puentes arbóreos, con una duración de dos sesiones, por lo que los resultados no generalizan a todas AFM N, ni a todos los contextos. Así mismo, esta secuencia didáctica representó la primera vez que el docente de la asignatura aplicó contenidos de EA, por lo que, dicha secuencia y las actividades pudieran mejorarse, en base a la experiencia. Tomando en cuenta los resultados obtenidos y las limitantes del estudio, surgen posibles líneas de investigación futuras. La primera, analizar el desempeño de los profesores en formación en actividades lúdicas con cuerdas durante su práctica real, en la sociedad. También surge la posibilidad de indagar la satisfacción del profesorado en forma ción utilizando técnicas e instrumentos cualitativos y cuantitativos.

\section{Referencias}

Agostini, F. Minelli, M., \& Mandolesi, R. (2018). O utdoor Education in Italian Kindergartens: How teachers perceive child developmental trajectories. Frontiers in Psychology, 9, 1-12. doi: 10.3389/ fpsyg 2018.01911

Badau, A. (2016). Aquagym.Theory and methodology. University Publish House.

Badau, D. (2017).Theeducational impact of implementation the education through adventure discipline in physical education and sports academic curriculum. Physical education of students, (3), 108-115. doi: 10.15561/ 20755279.2017.0302

BæenaExtremera, A. (2011). Programas didácticos para Educación Física a través de la Educación de Aventura. Espiral. Cuadernosdel Profesorado, 4 (7), 3-13. doi: 10.25115/ ecp.v4i7.914

Baena-Extremera, A., Granero-Gallegos, A., \& del Mar Ortiz-Camacho, M. (2012). Q uasi-experimental study of the effect of an adventure education programme on classroom satisfaction, physical self- concept and social goalsin physical education. Psychologica Belgica, 52 (4), 369386. doi: $10.5334 /$ pb-52-4-369

Barrable, A., \& Lakin, L. (2019). Nature relatedness in student teachers, perceived competence and willingness to teach outdoors: an empirical study. Journal of Adventure
Education and Outdoor Learning, 20(3), 189-201. doi 10.1080/ 14729679.2019.1609999

Baker, M. \& O 'Brien. W. (2019): Rethinking outdoor leadership: An Investigation of Affective Abilities in Australian Higher Education curriculum. Journal of AdventureEducation and Outdoor Learning, 20(3), 202-216. doi: 10.1080/ 14729679.2019.1634598

Caballero, P., Hernández-Hernández, E., \& Reina, M. (2018). A nálisis de los factores universales de las actividadesfísicas en el medio natural / actividades físicas de aventura en lanaturaleza: estudio preliminar. Espiral. Cuadernos del Profesorado, 11(22), 61-68. doi:10.25115/ ecp.v11i22. 1918

Caballero, P., Velo, C., \& Hernández, E. (2020). Desarrollo de competencias profesionales y personales a través de un programa basado en la pedagogía de la aventura en alumnado universitario. En J.C. Escaravajal, J. López, R. Baños, R. (Eds), Actividades en el Medio N atural y Deportes de Aventura (pp. 33-48). España: Asociación Andaluza de docencia e investigación en $\mathrm{EF}$.

Campos, G., \& Martínez, N. E. (2012). La observación, un método parael estudio delarealidad. Xihmai, 7(13), 4560.

Dalmau, J., Jiménez, R., Gómez, N., \& Gargallo, E. (2019). Diagnóstico escolar sobre el tratamiento de las activida des físicas en el medio natural dentro de la asignatura de Educación Física. Retos N uevasTendencias en Educación Física, Deporte y Recreación, 37, 460-464. doi: 10.47197/ retos. v37i37.71010

Doering, A. (2006). Adventure Learning: Transformative hybrid online education. Distance Education, 27 (2), 197215. doi: $10.1080 / 01587910600789571$

Escaravaja-Rodríguez, J. C., BæenaExtremera, A., Ayala Jiménez, J. D., \& Ruiz-Fernández, Z. M. (2017). Propuesta de raid de aventura escolar en Educación Física. Dilemas Contemporáneos: Educación, Política yValores 5(1).

Fernández-Río, J., Calderón, A., Hortigüela, D., PérezPueyo, A. \& Aznar, M. (2016). M odel os pedagógicos en educación física: consideraciones teórico-prácticas para docentes. Revista Española de Educación Física y Deportes, 413, 55-75.

Fuentesa-García, J., \& Zamorano-Sande, D. (2021). Incorporación de contenidos de actividades en el medio natural y de ocio en la educación formal (TSEAS): Una experiencia de espeleología. Retos N uevasTendencias en Educación Física, Deporte y Recreación, 39, 820-828. doi: 10.47197/ retos. v0i39.80157

Gilbertson, K., Bates, T., M cLaughlin,T., \& Ewert,A. (2006). Outdoor education: methods and strategies. EU: Human Kinetics.

Glaser, B. (1992). Emergence v Forcing Basics of GroundedT heory 
Analysis. EU: Sociology Press.

Gleason, M. A., \& Rubio, J. E. (2020). Implementación del aprendizaje experiencial en la universidad, sus beneficios en el alumnado y el rol docente. Revista Educación, 44(2), 279-298. doi: 10.15517/ revedu.v44i2.40197

González, R. A. , Zueck, M. D. C., Marín, R., \& Blanco, H. (2019). El sector turístico como un campo laboral para el educador físico: Revisión sistematizada. EmásF: revista digital de educación física, (58), 121-130.

Granero, A. (2015). Una experiencia de teleformación del profesorado de Educación Física (Primaria y Secunda ria) en actividades físicas en el medio natural. Retos, NuevasTendencias en Educación Física, D eporte y Recreación, 13, 39-45. doi: 10.47197/ retos. v0i13.35026

Guzmán, I., \& M arín, R. (2011). Lacompetenciay lascompetencias docentes: reflexiones sobre el concepto y la evaluación. Revista electrónica interuniversitaria de formación del profesorado, 36 (14-1), 151-163.

Hernández-Amezcua, H. A. , \& Martínez-Flores, A. (2017). Construcción de competencias didácticas por los estudiantes de la licenciatura en Educación Física. Kinesis Re vistaVeracruzana de Investigación Docente, 2(2), 44-59.

Hurtado-Barroso, M., Sanabrias-M oreno, D., Sánchez-Za fra, M., \& Cachón, J. (2020). Actividades Físicas en el Medio Natura: incidencia en la formación del Profesorado y su enseñanza en la ESO. Sportis, 6(1), 18-42. doi:10.17979/ sportis 2020.6.1.5769

Kalungwizi,V. J., Krogh, E., Gjøtterud, S. M., \& Mattee, A. (2018). Experiential strategies and learning in environmental education: lessonsfrom ateacher training college in Tanzania. Journal of Adventure Education and Outdoor Learning, 20(2), 95-110. doi: 10.1080/ 14729679.2018.1555047

Lamoneda, J., González-Víllora, S., \& Fernández-Río, J. (2020). Hibridando el A prendizaje Cooperđivo, laEducación Aventura y la Gamificación a través de la carrera de orientación. Retos, NuevasTendencias en Educación Físca, Deportey Recreación, 38, 754-760.

Leather, M., \& Nicholls, F. (2016). More than activities: using a'sense of place'to enrich student experience in adventure sport. Sport, Education and Society, 21(3), 443464. doi: 10.1080/ 13573322.2014.927758

Lee, J., \& Zhang, T. (2019). The Impact of Adventure Education on Students' Learning 0 utcomes in Physical Education: A Systematic Review. JTRM in Kinesiology. (18), 23-32.

Li, H. C., Chung, O. K. J., Ho, K.Y., Chiu, S.Y., \& Lopez, V. (2013). Effectiveness of an integrated adventure-based training and health education program in promoting physical activity among childhood cancer survivors.
Psycho-Oncology,22, 2601-2610. doi: 10.1002/ pon.3326

Li,W. H., Chung, J. O. \& Ho, E. K. (2013). Effectiveness of an adventure-based training programme in promoting the psychological well-being of primary schoolchildren. Journal of Health Psychology, 18(11), 1478-1492. doi: $10.1177 / 1359105312465102$

Losada, S. G. , \& García, A.T. (2018). Lasestrategiasdidácticas en la práctica docente universitaria. Profesorado, Revista de Curríaulum y Formación del Profesorado, 22(2), 371-388. doi: 10.30827/ profesorado.v22i2.7728

Lund, J., \& Tannehill, D. (2014). Standards-based physical education curriculum development. Jones and Bartlett.

Mackenzie, S. H., Son, J. S. \& Eitel, K. (2018). U sing outdoor adventure to enhance intrinsic motivation and engagement in science and physical activity: An exploratory study. Journal of outdoor recreation and tourism, 21, 76-86. doi 10.1016/ j.jort.2018.01.008

Maldonado, J., E. (2018). M etodología de la investigación social paradigmas cuantitativo, sociocrítico, cualitativo, complementario. Ediciones de laU.

Marinho, A. , Santos, P. M., Manfroi, M. N., Figueiredo, J. P., \& Brasil, V. Z. (2017). Reflections about outdoor adventure sports and professional competencies of physical education students. Journal of Adventure Education And Outdoor Learning, 17(1), 38-54. doi: 10.1080/ 14729679.2016.1218781

Martín, L., \& M ediavilla, L. (2020). Diferencias de género en el perfil y los hábitos de practicantes de actividades en el medio natural. Retos, NuevasTendencias en Educación Físca, Deportey Recreación, 38, 713-718. doi: 10.47197| retos. v38i38.78499

Mediavilla, L., Gómez, V., \& Gómez, V. (2020a). El medio natural y la metodología experiencial como recursos educativos, Ágora para la Educación Física y el Deporte, 22, 220-234. doi: 10.24197/ æfd. 0.2020.220-234

Mediavilla, L., Gómez,V., Martín, L., \& Gómez,V. (2020b). Las actividades en el medio natura y la metodología experiencial como herramientas para el desarrollo integral de los individuos Espiral, cuadernos del profesorado, 13(27), 134-143.

Merriam, S. (1998). Qual itativeresearch and casestudy applications in education. EU: Jossey-Bass.

Nascimento, J. V., Ramos, V., Marcon, D., Saad, M. A., \& Collet, C. (2009). Formação acadêmica e intervenção pedagógica nos esportes Motriz, 15(2), 358-366.

Navarro, R. , Arufe, V., \& Sancosmed, E. (2015). Las actividades en el medio natural en la educación física escolar. Formación y actitud del profesorado de Educación Primaria. Retos, N uevasTendencias en Educación Física, Deportey Recreación, 27, 122-126. doi: 10.47197/ retos. V0i27.34361 
Navarro-Patón, R., Rodríguez, J. E., \& Rico-Díaz, J. (2019). Formación defuturosmaestros de educación físicaapartir del aprendizaje experiencia. Sportis, 5(3), 423-443. doi: 10.17979/ sportis.2019.5.3.5466

Orellana, D. M., \& Sánchez, M. (2006). Técnicas de recolección de datos en entornos virtuales más usadas en la investigación cualitativa. Revisa de Investigación Educativa, (24), 205-222.

Orson, C. N., M cGovern, G., \& Larson, R.W. (2020). How challenges and peers contribute to social-emotional learning in outdoor adventure education programs. Journal of adolescence, 81, 7-18. doi: 10.1016/ j. adolescence. 2020.02.014

Pérez, M., Vilanova, A., \& Grimaldi, M. (2016). M ercado actual de trabajo de los graduados en Ciencias de laActividad Física y el Deporte: Mirada hacia el autoempleo. Journal of Sports Economics\& Management, 3(6), 149-162.

Rhonke, K. (1989). Cowstails and Cobras II. EU: Kendall/ Hunt.

Richmond, D., Sibthorp, J., Gookin, J., Annarella, S., \& Ferri, S. (2018). Complementing classroom learning through outdoor adventure education: O ut-of-schooltime experiences that make a difference. Journal of Adventure Education and Outdoor Learning, 18(1), 36-52. doi: 10.1080/ 14729679.2017.1324313

Rubio, C. F., \& Rico, E. R. (2012). Construcción de estructurasdecuerda; suaplicación recreativo-educativa، Emás: Revista digital de educación físca, (19), 77-98.

Sáez-Padilla, J., Tornero, I., \& Sierra, A. (2017). La situación actual de la formación en actividades en el medio natural según el profesorado deEducación Física. Un estudio cualitativo con expertos en Andalucía. Espiral. Cuadernos del Profesorado, 10(21), 100-117. doi: 10.25115/ ecp.v10i21.1033

San Martín, D. (2014). Teoría fundamentada y Atlas, ti: recursos metodológicos para la investigación educativa. Revisa Electrónica delnvestigación Educativa, 16(1), 104-122.

Sánchez, A. J., García, J., Grimaldi, M., \& Feria, A. (2017). 0 portunidades de emprendimiento: Evaluación de las tendencias relacionadas con la actividad física y el deporte del ACSM. Journal of Sports Economics \& M anagement, 7 (1), 2-13.

Scarf, D., Hayhurst, J. G. , Riordan, B. C., Boyes, M., Ruffman, T., \& Hunter, J. A. (2017). Increasing resilience in adolescents: The importance of social connectedness in adventure education programmes. Australasian Psychiatry, 25(2), 154-156. doi: 10.1177/ 1039856216671668

Stewart, K. , Carreau, J., \& Bruner, M. (2016). Team building using a challenge ropes course experience in youth sport. Revue phénEPS/ PHEnex Journal, 7(3).
Stigler, J. W., Gonzales, P., Kawanaka, T., Knoll, S. \& Serra no, A. (1999). The TIMSS videotape classroom study: Methods and findings from an exploratory research project on eighth-grade mathematics instruction in Germany, Japan, and the U nited States. Education Statistics Quarterly,1(2), 109-112.

Stuhr, P., Sutherland, S., Ressler, . ., \& O rtiz-stuhr, E. (2015). Students' perception of relationship skills during an adventure-based learning unit within physica education. Australian Journal of Outdoor Education, 18(1), 27-38. doi:10.1007/ BF03400977

Sutherland, S., \& Legge, M. (2016). The possibilities of «doing» outdoor and/ or adventure education in physical education/ teacher education. Journal of Teaching in Physical Education, 35(4), 299-312. doi: 10.1123/ jtpe. 2016-0161 Valencia, R., Salazar, C., Lozano, E. G., Figueroa, A., \& Flores, P. (2012). La recreación en el curriculum de los profesionales de educación física y deporte en México. Revista Latinoamericana de Recreación, 1(2).

Warren, K., Mitten, D., D’Amore, C., \& Lotz, E. (2019). Thegendered hidden curriculum of adventureeducation. Journal of Experiential Education, 42(2), 140-154. doi: 10.1177/ 1053825918813398

W illiams, A., \&Wainwright, N. (2016). A new pedagogical model for adventure in the curriculum: part oneadvocating for the model. Physical Education and Sport Pedagogy, 21(5), 481-500. doi: 10.1080/ 17408989.2015.1048211

W illiams, A., \& Wainwright, N. (2020) Re-thinking adventurousactivitiesin physical education: models-based approaches. Journal of Adventure Education and Outdoor Learning, 20(3), 217-229. doi:10.1080/ 14729679.2019 .1634599

Yin, R. (1994). Casestudy research: design and methods (2daed.). EU: SAGE.

Zueck, C., Guerrero, A., \& Blanco, H. (2009). Reforma curricular de la licenciatura en educación física: Evolución histórica al 2008. México: Universidad Autónoma de Chihuahua.

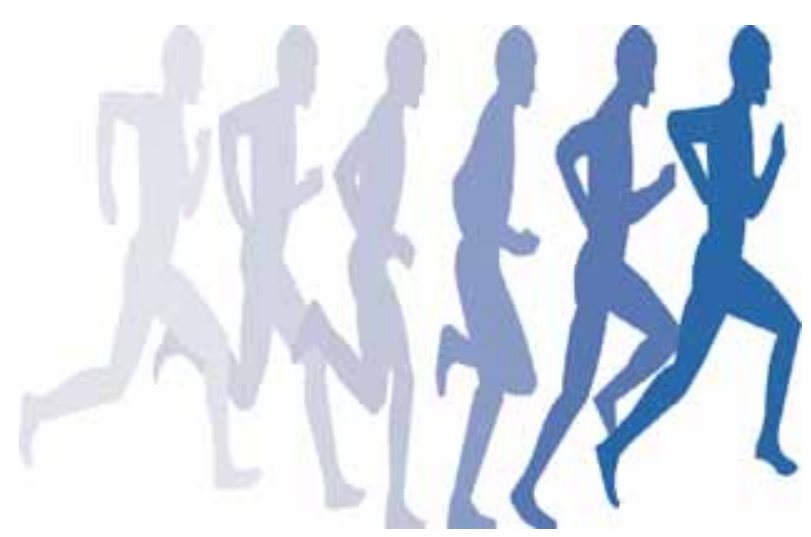

